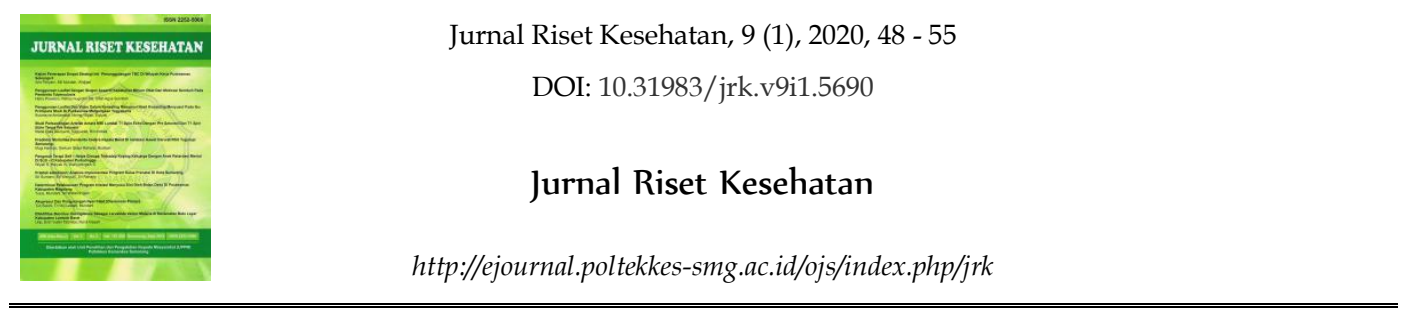

\title{
SEQUENCE APPLICATION OF BRAIN MRI WITH ORTHODONTIC BRACKET
}

\author{
Aryadiva N. Prayoga $\left.{ }^{a *}\right)$, Hermien Nugraheni ${ }^{b}$, Diyah Fatmasaric \\ a Universitas Aisyiyah; \\ Jl. Ringroad Barat No.63 Mlangi, Nogotirto, Gamping ,Sleman 55592, Indonesia \\ $b, c$ Poltekkes Kemenkes Semarang; \\ Jl. Tirto Agung, Pedalangan, Banyumanik, Semarang 50239, Indonesia
}

\begin{abstract}
Magnetic Resonance Imaging (MRI) examination of the brain at high resolution will be able to detect abnormalities in the brain that were not detected before. The MRI machine is equipped with a very strong magnetic force, therefore metal objects can interfere with the workings of the machine. Some patients may have an unnoticed magnet in the body (for example: orthodontic bracket). The purpose of this study is to analyze the effectiveness of sequences to reduce metal artifacts due to the installation of the Orthodontic Bracket. This type of research is a quantitative analytic with a quasi-experimental research design. The research design used was a one group pretest-posttest design to determine the optimization of the application of the Slice Encoding Metal Artifact Correction (SEMAC) and View Angle Tilting (VAT) sequences on Brain MRI with Orthodontic Bracket. Data analysis was performed with the Kruskal-Wallis H Test. There was an influence on the application of SEMAC and VAT on the anatomical brain MRI image information on overall anatomy with a $\mathrm{p}$ value of $0,047(\mathrm{p}<0,05)$. SEMAC sequences combined with T2 TSE are able to reduce metal artifacts well. VAT sequences combined with T2 TSE are able to reduce metal artifacts quite well.
\end{abstract}

Keywords : Brain MR; Orthodontic Bracket

\section{Introduction}

Magnetic Resonance Imaging (MRI) is a scanning procedure that uses a strong magnetic field and radio frequency to produce a more detailed image of the inside of the body. This medical examination is different from CT scans that use X-rays, because MRI does not use radiation that is potentially dangerous. (Brown, 2003)

Brain MRI is the diagnostic test most commonly used to detect brain tumors and diagnose brain cancer. This examination works by placing the patient in a magnetic field and using radio frequency energy to take image of the brain inside the head. MRI is a diagnostic examination that is more sophisticated than a normal X-ray examination, ultrasound, or CT (computer tomography) because it can provide

*) Corresponding Author (Aryadiva N. Prayoga)

E-mail: divaprayoga988@gmail.com extensive information that cannot be given by other imaging tests. (Edmund, 2017)

When undergoing Brain MRI, patients will be asked to lie in a special scanning machine with strong magnetic bore. MRI machines are equipped with very strong magnetic bore, therefore metal objects can interfere with the way the machine works and the results of procedures. All metal objects such as jewelry, watches, hair accessories, and even hearing aids or dentures must be removed to avoid magnetic interference. But in some patients, there may be an unnoticed magnet in the body, for example: braces (orthodontic bracket) (Chen et.al, 2011).

Orthodontic bracket is one of the tools to overcome tooth structure abnormalities that cause complaints. Conventional braces are made of stainless steel that are attached to the front of the teeth using special cement. (Chen et.al, 2011)

The use of MRI modalities has a vital role in the diagnosis of pathology which requires an 
image with good tissue contrast. MRI provides higher resolution and greater sensitivity to tissue structure and water content in the imaging field, which makes it possible to detect small changes in anatomy and vascular associated with cognitive impairment and dementia.(Brown, 2003)

With the increasing use of MRI in diagnostic imaging, developments and innovations in modality and MRI techniques are carried out to increase the sensitivity and specificity of modalities in diagnosing pathology. Brain MRI examination at high resolution will be able to detect abnormalities in the brain that were not detected before. (Ontario, 2014), (Edmund, 2017)

Another thing in the MRI modality that continues to be developed is the ability to reduce artifacts on examination.(Vernooij, 2007) Artifacts are undesirable images when pixels do not properly represent anatomical features. Artifacts occur because the underlying anatomy is visualized with inappropriate signals. There are several types of artifacts that can appear in MRI imaging, namely Phase Mismapping (motion), Aliasing (Wrap), Chemical Shift, Chemical Misregistration, Truncation, to artifacts due to Magnetic Susceptibility. (Catherine, 2014) (Poorsattar, 2016)

Vulnerability to magnetic is due to all magnetic networks differing degrees in magnetic characteristics. (Catherine, 2014) (Poorsattar, 2016) This results in differences in the frequency and phase of precession respectively. Phase differences cause dephasing at the boundaries of structures with very different magnetic susceptibility, and cause signal loss. Artifacts due to Magnetic Susceptibility can occur on any MRI examination with the use of metal implants in the anatomy examined, one of which is on an MRI examination of the Brain with an Orthodontic Bracket. (Reichert, 2015)( Filli, 2017)

The use of orthodontic brackets with metal content will cause signal changes in the related bracket and the surrounding tissue. These artifacts can damage the information contained in the image right at the most important part of an organ where the location is near a metal device. Metal artifacts arise due to vulnerability of local inhomogeneity of the tissue structure around the location of the presence of metal implants.(Woodward, 2000) Emptying the intravoxel decreases the received signal and produces an area of hypointense on the MRI image. (Woodward, 2000) (Moeller, 2010) Consequently, a frequency shift occurs due to misregistration of the spatial which is displayed as a bright and dark area in the image and geometric distortion occurs in the surrounding anatomy. To correct the signal vacancy and geometric distortion that occurs, it takes a technique capable of reducing artifacts caused by metal artifacts.

In addition to optimizing parameters in standard sequences, a number of specific sequence strategies have been developed to effectively reduce metal artifacts, one of which is the application of Slice Encoding Metal Artifact Correction (SEMAC) and View Angle Tilting (VAT). (Friedrich, 2016) (Jungmann, et al, 2015)(Chen et.al, 2011) SEMAC technique involves an additional phase coding step towards the slice to resolve geometric distortion along the slice. During image reconstruction, this information is used to sort distorted pixels into the correct pieces position, so that thorough field distortion can be corrected. Another technique is to combine SEMAC with VAT which allows better correction of the field artifacts from metal implants.(Faller, 2004)

Unlike SEMAC, the VAT technique uses an additional gradient in the direction of the slice selection during gradient reading. So the pixel image looks as if it is seen from an angle. The number of frequency artifacts shifts in the associated slice and in the direction of the frequency in which it is skewed. By looking at it from an oblique angle, the received signal can be projected to the pixel position corrected from the image matrix, so the artifacts of the metal implant can be corrected. (Westbrook, 2014) (Moore, 2014) ( Ellis, 2006)

The purpose of this study is to analyze the effectiveness of the application of Slice Encoding Metal Artifact Correction (SEMAC) and View Angle Tilting (VAT) to reduce metal artifacts on brain MRI examination with Orthodontic Bracket.

\section{Method}

This research type is a quantitative analytic research with a quasi-experimental research design. The research design used was a one group pretest-posttest design to determine the optimization of the application of the Slice Encoding Metal Artifact Correction (SEMAC) and View Angle Tilting (VAT) sequences on 
Brain MRI with Orthodontic Bracket. The populations in this study were volunteers who used the Orthodontic Bracket and performed an Brain MRI examination at Pusat Otak Nasional (PON) Hospital Jakarta.

The inclusion criteria of this study were: healthy volunteers with Orthodontic Bracket (Stainless steel brackets, Titanium brackets, Ceramic brackets); BMI (18.5-22.9), age range 1535 years. The sampling technique in this study was a purposive sampling.

Data analysis was performed with the Kruskal-Wallis H Test, with the aim was to see whether or not there were differences in influence between treatments of anatomical information including: clarity of organs in the Temporo mandibular joints, Oral Cavity, Posterior Cerebral Fossa, and Maxillary Sinus regions.

\section{Result and Discussion}

In research on the effect of applying SEMAC and VAT T2 TSE sequences to reduce metal artifacts on brain MRI examination with an axial section Orthodontic Bracket, the following results are obtained:

The suitability test results show that there was a very good perception agreement between respondent 1 and respondent 2 with a $p$ value of 0,907 .

Table 1. The respondent suitability test results

\begin{tabular}{cc}
\hline respondent suitability test & p value \\
\hline respondent 1 and respondent 2 & 0,907 \\
\hline
\end{tabular}

To determine the effect of applying T2 TSE, T2 TSE SEMAC, and T2 TSE VAT sequences on Brain MRI examination with Orthodontic Bracket can be seen in table 2 .

Table 2. Influence Test Results from Brain MRI Anatomy

\begin{tabular}{ccrcc}
\hline $\begin{array}{c}\text { Anatomy } \\
\text { Information }\end{array}$ & Var & $\begin{array}{c}\text { Mean } \\
\text { Rank }\end{array}$ & N & $\begin{array}{c}\text { p } \\
\text { value }\end{array}$ \\
\hline Brain MRI & TSE & 5,69 & 8 & \\
Anatomy & VAT & 15,19 & 8 & 0,003 \\
& SEMAC & 16,63 & 8 & \\
\hline
\end{tabular}

Table 2 shows the effect of applying SEMAC and VAT to the Brain MRI anatomical image information with a $\mathrm{p}$ value of 0.003 ( $\mathrm{p}$
$<0.05)$. From the Mean Rank table, it is known that SEMAC was higher than other variations of 16.63 while in the application of VAT the mean rank was 15.19 and the lowest mean TSE obtained was 5.69. Furthermore, the effect of SEMAC and VAT application on each organ were tested to determine differences in anatomic information from Oral cavity, temporo mandibular joint, posterior cerebral fossa, and maxillary sinus.

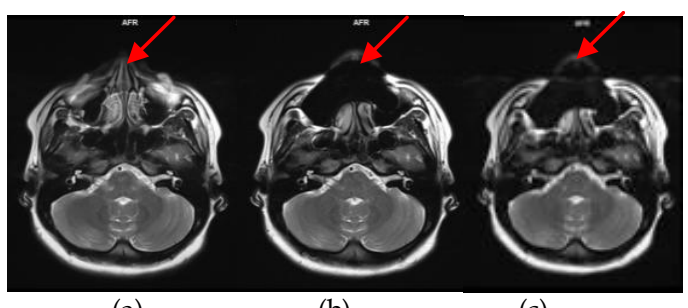

(a)

(b)

(c)

Figure 1. Image Results Effects of the

Application of Metal Reduction Brain MRI in Oral Cavity Organs. (a) T2 TSE SEMAC, (b) T2 TSE VAT, (c) T2 TSE

Table 3. Test Results Effect of the Application of Metal Artifacts on Oral Cavity organs

\begin{tabular}{ccccc}
\hline $\begin{array}{c}\text { Anatomy } \\
\text { Information }\end{array}$ & Var & $\begin{array}{c}\text { Mean } \\
\text { Rank }\end{array}$ & N & p value \\
\hline \multirow{3}{*}{ Oral Cavity } & TSE & 5,50 & 8 & 0,001 \\
& VAT & 12,63 & 8 & 0,001 \\
& SEMAC & 19,38 & 8 & \\
\hline
\end{tabular}

Table 3 shows the influence of the application of SEMAC and VAT to the MRI anatomical image information of the Oral Cavity organ with a $p$ value of $0,000(p<0.05)$. From the Mean Rank table, it is known that the variation of high as high as 19.38 occurs in the application of SEMAC, while in the application of VAT the variation is 12.63 and the lowest variation of the TSE is 5.50 .

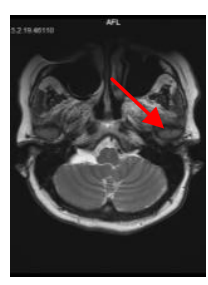

(a)

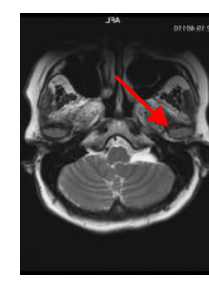

(b)

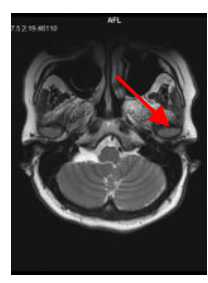

(c)
Figure 2. Image Results Effects of the Application of Metal Reduction Brain MRI in Temporo mandibular Joint organ. (a) T2 TSE SEMAC, (b) T2 TSE VAT, (c) T2 TSE 
Table 4 Test Results Effect of the Application of Metal Artifacts on temporo mandibular Joint organ

\begin{tabular}{ccrcc}
\hline $\begin{array}{c}\text { Anatomy } \\
\text { Information }\end{array}$ & Var & $\begin{array}{r}\text { Mean } \\
\text { Rank }\end{array}$ & N & p value \\
\hline temporo & TSE & 5,50 & 8 & \\
mandibular & VAT & 13,00 & 8 & 0,001 \\
joint & SEMAC & 19,00 & 8 & \\
\hline
\end{tabular}

Based on table 4, it is seen that there was an effect of SEMAC and VAT application on the anatomical image information of the Brain MRI in the temporo mandibular joint organ with a $\mathrm{p}$ value of $0,000(p<0.05)$. It is known that the highest SEMAC variation was 19.00 while in the VAT application the variation was 13.00 and the lowest TSE variation was 5.50.

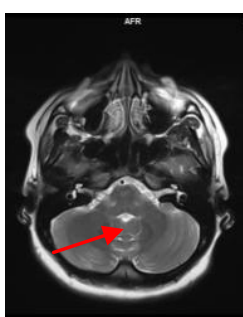

(a)

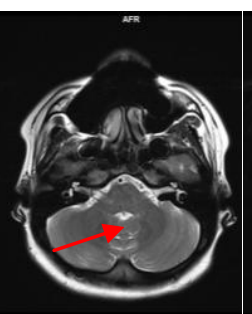

(b)

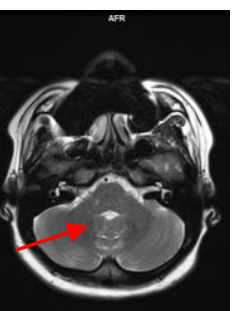

(c)
Figure 3. Image Results Effects of the Application of Metal Reduction Brain MRI in Posterior Cerebral Fossa organ (a) T2 TSE SEMAC, (b) T2 TSE VAT, (c) T2 TSE

Table 5. Test Results Effect of the Application of Metal Artifacts on Posterior Cerebral Fossa organ

\begin{tabular}{ccrcc}
\hline $\begin{array}{c}\text { Anatomy } \\
\text { Information }\end{array}$ & Var & $\begin{array}{r}\text { Mean } \\
\text { Rank }\end{array}$ & N & p value \\
\hline Posterior & TSE & 9,00 & 8 & \\
Cerebral Fossa & VAT & 11,56 & 8 & 0,047 \\
& SEMAC & 16,94 & 8 & \\
\hline
\end{tabular}

Based on table 5, it can be seen that there was an influence on the application of SEMAC and VAT to the Brain MRI anatomical image information in the Posterior Cerebral Fossa organ with a $\mathrm{p}$ value of $0.047(\mathrm{p}<0.05)$. From the Mean Rank table, it is known that SEMAC was higher than other variations by 16.94 while in the VAT application the mean rank was 11.56 and the lowest mean TSE obtained rank was 9.00 .

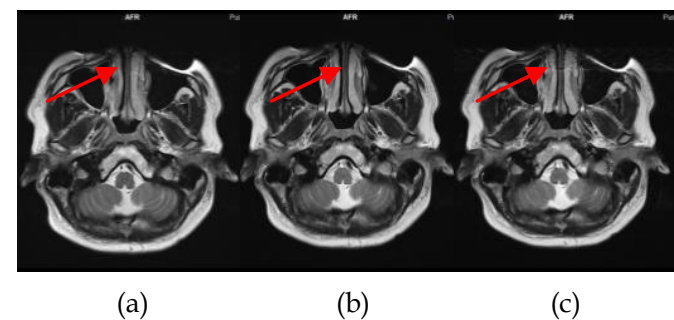

Figure 4. Image Results Effects of the Application of Metal Reduction in Brain MRI Maxillary Sinus organ (a) T2 TSE SEMAC, (b) T2 TSE VAT, (c) T2 TSE

Table 6 Test Results Effect of the Application of Metal Artifacts on Maxillary Sinus organ

\begin{tabular}{ccccc}
\hline $\begin{array}{c}\text { Anatomy } \\
\text { Information }\end{array}$ & Var & $\begin{array}{c}\text { Mean } \\
\text { Rank }\end{array}$ & N & p value \\
\hline Maxillary & TSE & 6,88 & 8 & \\
Sinus & VAT & 15,88 & 8 & 0,011 \\
& SEMAC & 14,75 & 8 & \\
\hline
\end{tabular}

Based on table 6, it can be seen that there was an influence on the application of SEMAC and VAT to the MRI Brain anatomical image information on Maxillary Sinus organs with a $p$ value of 0.011 ( $p<0.05)$. From the Mean Rank table, it is known that the VAT was higher than the other variations of 15.88 while in the application of SEMAC the mean rank was 14.75 and the lowest mean TSE obtained was 6.88 .

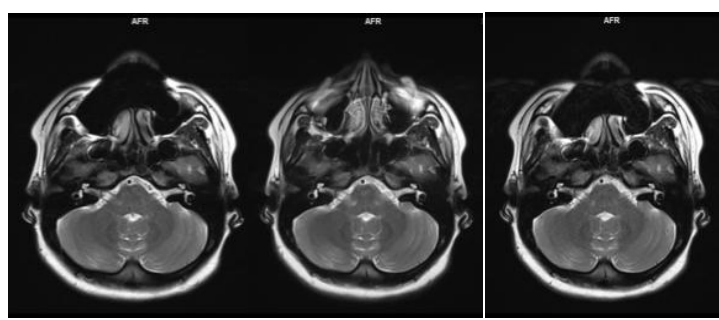

(a)

(b)

(c)

Figure 5. Image Results Effects of the Application of Metal Reduction MRI Brain on overall anatomy (a) T2 TSE SEMAC, (b) T2 TSE VAT, (c) T2 TSE

Table 7. Test Results Effect of the Application of Metal Artifacts on overall anatomy organ

\begin{tabular}{clrrc}
\hline $\begin{array}{c}\text { Anatomy } \\
\text { Information }\end{array}$ & Var & $\begin{array}{c}\text { Mean } \\
\text { Rank }\end{array}$ & N & p value \\
\hline \multirow{2}{*}{ overall } & TSE & 8,50 & 8 & \\
anatomy & VAT & 16,00 & 8 & 0,047 \\
& SEMAC & 13,00 & 8 & \\
\hline
\end{tabular}


Based on table 7, it can be seen that there was an influence on the application of SEMAC and VAT on the anatomical brain MRI image information on overall anatomy with a $p$ value of 0.047 ( $p$ <0.05). From the Mean Rank table, it is known that the VAT was higher than the other variations of 16.00 while in the application of SEMAC the mean rank was 13.00 and the TSE mean rank was lowest at 8.50.

Based on the data analysis in table 2, it is found that the use of the SEMAC sequence had a higher value compared to other VAT and TI TSE sequences, with a mean rank value of 16.63 , in the brain MRI anatomy image information with axial pieces with the installation of Orthodontic Bracket.

Image quality is an important parameter that needs to be known by a radiographer to produce an optimal picture of the MRI image by knowing the factors that affect the quality of the MRI image image. (Brown, 2003) (Jungmann, et al, 2015) ( Ellis, 2006) Image quality on MRI is influenced by several factors, including SNR, CNR, spatial resolution and scan time. In addition, the presence of artifacts also greatly affects the quality of the image, especially the anatomical information that will be assessed and become the basis in determining the diagnosis. (Ontario, 2014)(Catherine, 2014)

Anatomic information is the result of observations from radiology specialists on the quality of images produced by MRI images. Qualitatively, the assessment is done by assessing anatomical clarity and image contrast information in general on brain MRI examination. This examination uses several variations of sequences that are useful for reducing metal artifacts, including T2 TSE SEMAC, T2 TSE VAT and T2 TSE axial pieces with anatomical criteria assessed include Oral cavity, temporo mandibular joint, posterior cerebral fossa, and maxillary sinus. (Suryanegara, 2000) Assessment of axial cuts anatomical information used a questionnaire, which were assessed to several respondents. In the questionnaire there was an assessment of numbers that had to be filled in by the respondent whether the anatomic information was not good, sufficient and good. In this study, anatomical information was measured on an ordinal scale.

From the respondent's assessment data, a Conformity Test was conducted to assess that respondent's assessments 1 and 2 had a strong reliability or interpretation and coefficient. To provide an interpretation of the coefficient and correlation can be stated with a value of $0.6-0.8$ meaning that it has a strong relationship and a value of $0.8-1$ meaning that it has a very strong relationship. The results of the Conformity Test in table 2 show that respondents 1 and 2 had a strong relationship, then the first respondent data were taken for the next statistical test. (Khan, 2015) (JADA, 2007)

In MRI of the head and neck region, the presence of ferromagnetic metals in some dental materials causes a non-homogeneous magnetic field. Metal-based materials give rise to individual magnetic fields and are able to change the frequency of proton precession in adjacent tissues. (Mamourian, 2010) (Reimer, 2010) This is able to inhibit signal formation and result in incorrect diagnosis.

Regarding the use of orthodotic brackets that can cause metal artifacts, and according to Shellock et al. in various safety-related studies conducting MRI with respect to 16 dental materials tested and devices containing ferromagnetic materials, it is found that only three samples (Stainlesss Steel, amalgam, and silver point) caused problems for patients, during MRI scanning, because the materials were magnetically activated. (Liney G, 2006) (Donald, 2006)

In another study, Okano et al. suggested that ceramic orthodontic brackets applied to anterior teeth and metal bonds to molar teeth does not have a direct effect on the diagnostic quality of MRI or on imaging the mandibular temporo joint. (Westbrook, 2014) Stainles steel material and other metals used in teeth fake creates a large number of artifacts that tend to blur the details of MRI images in the face area. (Simanjuntak, 2014)

The selection of parameters in the examination is also an important thing to consider because the selection of parameters is very influential on the results of the acquisition of imaging data. Each parameter section also has its own function in displaying image quality. (Smith, 2010) The parameters that become the control variables in the study are TR (Time Recovery), TE (Time Echo), SliceThickness, FOV, NEX, Matrix, Age, and BMI values. Because these variables are control variables, they will not affect or change the results of the study. (Edmund, 2017) (Westbrook, 2014) (Wellman, 2011)

Then, according to Morikuni et al, when conducting research to examine the appearance 
of metal artifacts caused by mounting brackets, MRI scanning of the oral cavity was done with Stainles steel bracket types. (Bachschmidt, 2012) Stainles steel brackets used in their study contained $98 \%$ ferromagnetic material and particles, and therefore had a large enough capacity to disturb the magnetic field gradient. (Catherine, 2014) (Bachschmidt, 2012) Likewise, in this study, we found out that brackets with stainles steel wire induced more metal artifacts.

Research by applying various types of brackets to see the influence of each bracekt on metal artifacts that appear on MRI images has never been done before.

SEMAC is a sequence based on 2D TSE. ${ }^{32}$ The addition of encoding dimensions is done to correct field distortion. Each slice is added to 3D encoding slice. This provides information on how the drawing of distorted slices, such as shifting signals perpendicular to the plane of the image can be corrected by postprocessing during image reconstruction. (Bachschmidt,2012) The main drawback of SEMAC is the increase in imaging time required. (Talbot, 2016)

The results show that the SEMAC sequence combined with T2 TSE was able to reduce metal artifacts well which was supported by high mean rank in the Kruskal-Wallis H. effect test. This result is consistent with research conducted by Chen, Christina et al confirming that SEMAC was good to reduce metal artifacts, providing high resolution images and good soft tissue contrast. Meanwhile, Jungmann, Pia et al stated that SEMAC is significant in reducing metal artifacts. (Smith, 2010) Clinical results suggest that this new technique would be useful for detecting postoperative periprostetic pathology. (Reimer, 2010) SEMAC sequences are able to reveal small structures in certain tissues compared to VAT, but the disadvantage of this sequence is that the longer time required for data acquisition if compared to other sequences. (Edmund, 2017) (Westbrook, 2014) (JADA, 2007)

VAT is a acquisition sequence scheme that is modified so that it can compensate for distortion in the aircraft. This sequence is applied to the Turbo Spin Echo (TSE) sequence. View Angle Tilting, or VAT, is intended for correction of chemical changes and induction of B0-inhomogeneity. The main idea is to apply slice selection gradients during data acquisition, which has the effect of tilting view angle with $\theta$ $=\tan -1$ where Gslice and Gfreq are selective gradient slices. In conventional SE acquisitions, during slice selection, all rotating turns tilt their frequency on a band (ie slice selection bandwidth). (Filli, 2017) (Moore, 2014) (Liney G, 2006) When the gradient is removed, the spin no longer has the same magnetic field due to inhomogeneities, which means that they no longer have the same Larmor frequency. (Friedrich B, et al, 2016) (Suryanegara, 2000) So they have the same Larmor frequency, during the encoding of the frequency, the same gradient is used for slice selection and is applied at the same time as the gradient reading (Suryanegara, 2000)

The results show that the VAT sequence combined with T2 TSE was able to reduce metal artifacts quite well, based on the mean rank value in the Kruskal-Wallis $H$. effect test. However, this application had experienced blurring effect on MRI images that emerged as a result of applying sequences VAT

This is consistent with the statement from Bachschmidt $\mathrm{T}$, that the effect that occurs is a field distortion along the direction of reading. VAT can cause image blurring caused by two separate effects. One reason is the shift in geometric slices. This effect can be reduced by using thin slices and high resolution. The second source of blurring is a low-pass filter that is superimposed by signal readings due to the addition of VAT gradients. This can be reduced by a short reading duration. (Talbot BS, et.al, 2016)

Of the three sequences used, the SEMAC sequence was considered the most effective in reducing the presence of metal artifacts arising from the installation of the Orthodontic Bracket on Brain MRI examination. Statistical results show that the mean rank value of the whole anatomy that appears in the SEMAC sequence is higher than the VAT or T2 TSE, although there are two parts of image information, namely the Maxillary Sinus organ and the anatomy as a whole states that VAT has a high mean rank value.

In this stud, it can be concluded that the application of SEMAC sequences on Brain MRI examination to reduce the presence of metal artifacts caused by the installation of Orthodontic Bracket is considered to have better anatomical information when compared to other sequences such as VAT and T2 TSE. (Jawad Z, et.al, 2015), (Noelia A, et.al, 2015), (Beau A, et.al, 2015). 


\section{Conclusion and Suggestion}

SEMAC sequences combined with T2 TSE are able to reduce metal artifacts well. VAT sequences combined with T2 TSE are able to reduce metal artifacts quite well, however, the mean rank generated is still below the T2 SEMAC value. This application also affects the MRI image that has blurred as a result of the application of the TSE TAT VAT sequence. A more optimal sequence to reduce metal artifacts is T2 TSE SEMAC, where the sequence is able to reveal thin structures that are not visible in the T2 TSE or T2 TSE VAT sequences. Further research needs to be done about the effect of each type of bracket on the artifacts that appears on the results of the MRI brain image examination with the Orthodontic Bracket.

\section{Acknowledgment}

Acknowledgments and highest appreciation go to Mrs. Fatimah Sugiyanto, S.ST, M.Kes and Dr. drg. Diyah Fatmasari, MDSc for ongoing support and encouragement.

\section{References}

Bachschmidt T, Lipps F, Ph D, Nittka M. syngo WARP - Metal Artifact Reduction Techniques in Magnetic Resonance Imaging. Magnetom Flash. 2012;(2):2425.

Beau A, Bossard D, Gebeile-Chauty S. Magnetic resonance imaging artefacts and fixed orthodontic attachments. Eur J Orthod. doi:10.1093/ejo/cju020

Brown, et.al. MRI Basic Principles and Applications. Vol 39.; 2003.

Chen CA, Chen W, Goodman SB, et al. New MR imaging methods for metallic implants in the knee: Artifact correction and clinical impact. J Magn Reson Imaging. $\quad$ 2011;33(5):1121-1127. doi:10.1002/jmri.22534

Edmund JM, Nyholm T. A review of substitute CT generation for MRI-only radiation therapy. Radiat Oncol. 2017;12(1):1-15. doi:10.1186/s13014-016-0747-y

Ellis H. Clinical Anatomy.; 2006. doi:10.1002/(SICI)10982353(1996)9:1<61: :AID-CA14>3.0.CO;2-8

Faller A, Schuenke M. The Human Body - An Introduction to Structure and Function. Vol 53.; 2004. doi:10.1017/СBO9781107415324.004
Filli L, Jud L, Luechinger R, et al. Materialdependent implant artifact reduction using SEMAC-VAT and MAVRIC: A prospective MRI phantom study. Invest Radiol. 2017; 52(6): 381-387. doi:10.1097/RLI.0000000000000351

Friedrich B, Wostrack M, Ringel F, et al. Novel Metal Artifact Reduction Techniques with Use of Slice-Encoding Metal Artifact Correction and View-Angle Tilting MR Imaging for Improved Visualization of Brain Tissue near Intracranial Aneurysm Clips. Clin Neuroradiol. 2016;26(1):31-37. doi:10.1007/s00062-014-0324-4

Haris Khan. Orthodontic Bracket Selection, Placement and Debodning.

JADA, Vol. 138 http://jada.ada.org April 2007. Braces Straighter teeth can improve oral health. Am Dent Assoc. 2007;30(9):504505. doi:10.1016/S0096-6347(44)90006-2

Jawad Z, Bates C, Hodge T. Who needs orthodontic treatment? Who gets it? and who wants it? Br Dent J. 2015;218(3):99103. doi:10.1038/sj.bdj.2015.51

Jungmann PM, Ganter C, Schaeffeler CJ, et al. View-angle tilting and slice-encoding metal artifact correction for artifact reduction in MRI: Experimental sequence optimization for orthopaedic tumor endoprostheses and clinical application. PLoS One. 2015;10(4):1-18. doi:10.1371/journal.pone.0124922

Liney G. MRI in Clinical Practice. Vol 39.; 2006. Mamourian, Alexander C. . Practical Mr Physics.; 2010.

Moeller TB. MRI Parameters and Positioning.; 2010.

Moore, Keith L Clinically Oriented Anatomy; 2014. doi:10.1016/B978-0-7020-43123.00031-3

Noelia A, Itati GL, Matilde S. The need for orthodontic treatment according to severity of malocclusion in adult patients. 2015.

Ontario HQ. The Appropriate Use of Neuroimaging in the Diagnostic WorkUp of Dementia: OHTAC Recommendation. 2014;14(1).

Poorsattar-Bejeh Mir A, Rahmati-Kamel M. Should the orthodontic brackets always be removed prior to magnetic resonance imaging (MRI)? J Oral Biol Craniofacial Res. 2016;6(2):142-152. doi:10.1016/j.jobcr.2015.08.007 
Reichert M, Ai T, Morelli JN, Nittka M, Attenberger U, Runge VM. 2015. Metal Artifact Reduction in MR Imaging at Both 1,5 $\mathrm{T}$ and 3T Using Slice-Encoding or Metal Artifact Correction and View Angle Tilting.

Reimer P. Clinical MR Imaging.; 2010. doi:10.1007/978-3-540-74504-4

Simanjuntak, Josepa ND. Studi Analisis Echo Train Length Dalam K- Space Serta Pengaruhnya Terhadap Kualitas Citra Pembobotan T2 Fse Pada MRI 1,5 T. 2014;17(1):7-12.

Smith TB, Nayak KS. MRI artifacts and correction strategies. Imaging Med. 2010;2(4):445-457doi:10.2217/iim.10.33

Suryanegara, Rina J Memperbaiki Dan Memperindah Posisi Gigi Anak.; 2000.

Talbot BS, Weinberg EP. MR Imaging with Metal-suppression Sequences for
Evaluation of Total Joint Arthroplasty. RadioGraphics. 2016;36(1):209-225. doi:10.1148/rg.2016150075

Vernooij MW, Ikram MA, Tanghe HL, et al. Incidental Findings on Brain MRI in the General Population. N Engl J Med. 2007;357(18):1821-1828. doi:10.1056/NEJMoa070972

Wellman F. Evaluation of methods for MR imaging near metallic hip prostheses. 2011:37. doi:10.1155/2012/815870

Westbrook, Catherine W. Handbook of MRI Technique 4th Edition. Vol 136.; 2014.

Westbrook C, Talbot J, Roth C. MRI: In Practice.; 2011.

Westbrook, Catherine W. MRI at a Glance. Vol 196.; 2002. doi:10.2214/AJR.10.6192

Woodward P. MRI for Technologist. Mc.GrawHill Medical; 2000. 